\title{
Atypical Findings on Magnetic Resonance Imaging in the Patients with Active Pyogenic Spondylitis in Japanese University Hospitals
}

\author{
Toshimi Aizawa, ${ }^{1}$ Hiroshi Ozawa, ${ }^{1}$ Tomoaki Koakutsu, ${ }^{1}$ Takeshi Nakamura, ${ }^{1}$ \\ Haruo Kanno, ${ }^{1}$ Toru Hirano, ${ }^{2}$ Atsuki Sano, ${ }^{2}{\text { Takui } \text { Ito }^{3} \text { and Eiji Itoi }}^{1}$ \\ ${ }^{1}$ Department of Orthopaedic Surgery, Tohoku University School of Medicine, Sendai, Miyagi, Japan \\ ${ }^{2}$ Department of Orthopaedic Surgery, Niigata University School of Medicine, Niigata, Niigata, Japan \\ ${ }^{3}$ Department of Orthopaedic Surgery, Niigata City General Hospital, Niigata, Niigata, Japan
}

\begin{abstract}
Recently, aging population and immuno-compromised patients have been rising in Japan. Accordingly, patients with pyogenic spondylitis have been increasing and may present atypical clinical features. University hospitals treat many elderly patients and patients with poor general condition. Therefore, patients with pyogenic spondylitis treated at two university hospitals were retrospectively investigated to clarify the recent clinical and radiologic characteristics of this infection. There were 30 patients (average age: 68 years) treated in two university hospitals between 2009 and 2010 . The onset was acute or subacute in 15 patients, insidious in 7 and unclassified in 8 . Culture tests were performed in 25 patients, and the causative microorganisms were identified in 20 patients with the identification rate of $80 \%$, including 4 patients infected by methicillin-resistant staphylococci. Classically, active pyogenic spondylitis is characterized by typical findings on magnetic resonance imaging (MRI): obvious signal decrease in T1-weighted image (WI) and increase in T2WI with contrast enhancement found in most of the bodies of two adjacent vertebrae and the intervening intervertebral disc. Among 29 patients with active pyogenic spondylitis, whose lesions were not in the healing stage, 16 patients demonstrated at least one of the atypical MRI findings; 9 patients showed involvement $\geq 3$ vertebrae or only 1 vertebra, 5 showed the signal changes of the lesions involving small, spotty, or faint areas, and 3 showed small vertebral lesions but larger epidural or paraspinal abscesses. In conclusion, currently, about half of the patients with pyogenic spondylitis demonstrate atypical MRI findings in the university hospitals in Japan.
\end{abstract}

Keywords: magnetic resonance imaging; pyogenic spine; spine radiography; spondylitis; university hospitals Tohoku J. Exp. Med., 2013 September, 231 (1), 13-19. (C) 2013 Tohoku University Medical Press

\section{Introduction}

Pyogenic spondylitis, a bacterial infection of the spine, is diagnosed on the basis of clinical, radiologic and microbiologic grounds. Among several radiological diagnostic modalities available, magnetic resonance imaging (MRI) has evolved to be most essential (Tsiodras and Falagas 2006). Experimentally, MRI can reveal positive changes 2 weeks after the actual onset of this disorder (Carragee 1997; $\mathrm{Kim}$ et al. 2011). The typical, classical signal pattern on MRI is a signal decrease in T1-weighted image (WI) and a signal increase in T2WI because of bone marrow edema, and contrast medium can reveal enhancement reflecting inflammation, which are usually found in much of the bodies of 2 adjacent vertebrae and the intervening intervertebral disc (Modic et al. 1985; An and Seldomridge 2006; DeSanto and Ross 2011; Cheung and Luk 2012; Leone et al. 2012).
The recent shift in demographics in Japan including an aging population, an increase in the number of patients with chronic debilitating disorders (immuno-compromised hosts), and the development and widespread use of immuno-suppressive agents might have affected the change in the clinical features of pyogenic spondylitis (Yoshimoto et al. 2011; Fukuda et al. 2012). For example, pyogenic spondylitis was once most frequently observed in adolescents to middle-aged individuals; however, it is currently most prevalent in the elderly (Yoshimoto et al. 2011). A decline in the acute-onset type, which is marked by high fever and severe back pain, and an increase in the insidiousonset type, where the exact time of onset is unknown, have been noted (Kulowski 1936; Ozawa 2008). Also, cases of methicillin-resistant Staphylococcus aureus (MRSA) spondylitis have been increasing, especially among high-risk elderly patients (Washio et al. 1997).

The demographic shift in patients may also affect

Received May 13, 2013; revised and accepted August 8, 2013. Published online August 31, 2013; doi: 10.1620/tjem.231.13.

Correspondence: Toshimi Aizawa, M.D., Department of Orthopaedic Surgery, Tohoku University School of Medicine, 1-1, Seiryo-machi, Aoba-ku, Sendai, Miyagi 980-8574, Japan.

e-mail: toshi-7@ra2.so-net.ne.jp 
anticipated radiologic findings. However, to our knowledge, there have been no studies till date indicating this possibility. Therefore, in the present study, we analyzed the MRI findings of patients with pyogenic spondylitis treated at two university hospitals between 2009 and 2010 in order to confirm whether these patients still demonstrate typical, classic MRI findings since many elderly patients and patients with severe general conditions such as immunocompromised hosts are treated in university hospitals in Japan.

\section{Materials and Methods}

The study protocol was approved by the Ethics Committee of Tohoku University School of Medicine.

\section{Patient Population}

Between 2009 and 2010, the medical records and imaging results of patients treated for pyogenic spondylitis at the Department of Orthopaedic Surgery, Tohoku University Hospital in Miyagi Prefecture and Niigata University Medical and Dental Hospital in Niigata Prefecture, were retrospectively extracted. A diagnosis of pyogenic spondylitis was made on the basis of patient's clinical history (including type and onset of symptoms), physical examination (including neurological findings), blood examination, radiologic assessments, and a positive microorganism culture from blood or spinal biopsy. Patients were also diagnosed as having pyogenic spondylitis if there were suggestive clinical findings, appropriate radiological features, negative culture examination from a suspected spinal lesion but showed elevated inflammatory markers, and a favorable response to antimicrobial therapy. Patients were confirmed to have no other inflammatory diseases such as urinary tract infection or gall bladder inflammation before a definitive diagnosis of pyogenic spondylitis was made. Patients infected by tuberculosis and mycosis were also excluded. In total, 30 patients were treated during the study period, which included 16 males and 14 females with a mean age of onset of 68 years (range, 36-83 years).

\section{Clinical Evaluation}

The following clinical data were collected from patient medical records: clinical history including onset type, white blood cell (WBC) count and C-reactive protein (CRP) at the initial visit, general condition of the patients, and the causative microorganisms. The onset type was classified using the modified Kulowski's classification (1936): 1) acute: onset with typical acute inflammatory symptoms such as high fever, severe back pain, and stiffness of the spinal column; 2) subacute: onset with slight fever and back pain; 3) insidious: unknown time of onset with only mild local symptoms such as back pain continuing for a certain period, without accompanying fever (Ozawa 2008; Yoshimoto et al. 2011); and 4) unclassified.

\section{Radiological Evaluation}

Plain radiographs and MRI from the patient's initial visit were evaluated. First, the spinal level and the number of vertebrae involved were confirmed. Then, plain radiograph findings were staged using the Griffiths and Jones classification (1971): 1) early stage: edema of the paravertebral tissues, narrowing of the intervertebral disc interval, and mild destruction around the vertebral endplates i.e., the top and bottom portions of the vertebral bodies interfacing with the intervertebral disc; 2) destructive stage: bone destruction, collapse of softened vertebral bodies, and bone proliferation that is a form of irregular excrescences of bones; and 3) healing stage: bone sclerosis and new bone formation.

On MRI, pyogenic spondylitis typically demonstrates an obvious decrease in the signal intensity on T1WI and increase in signal intensity on T2WI, and is diffusely and homogeneously enhanced with gadolinium (Gd) contrast, which is usually found in 2 adjacent vertebrae and the intervening intervertebral disc (Modic et al. 1985; An and Seldomridge 2006; DeSanto and Ross 2011; Cheung and Luk 2012; Leone et al. 2012) (Fig. 1). Those signal changes can be detected much of the vertebral bodies (Modic et al. 1985; Cheung and Luk 2012). We defined such findings as typical MRI findings of pyogenic spondylitis for the purpose of this study. After evaluating the data, the MRI findings of the patients were judged whether or not they were typical.

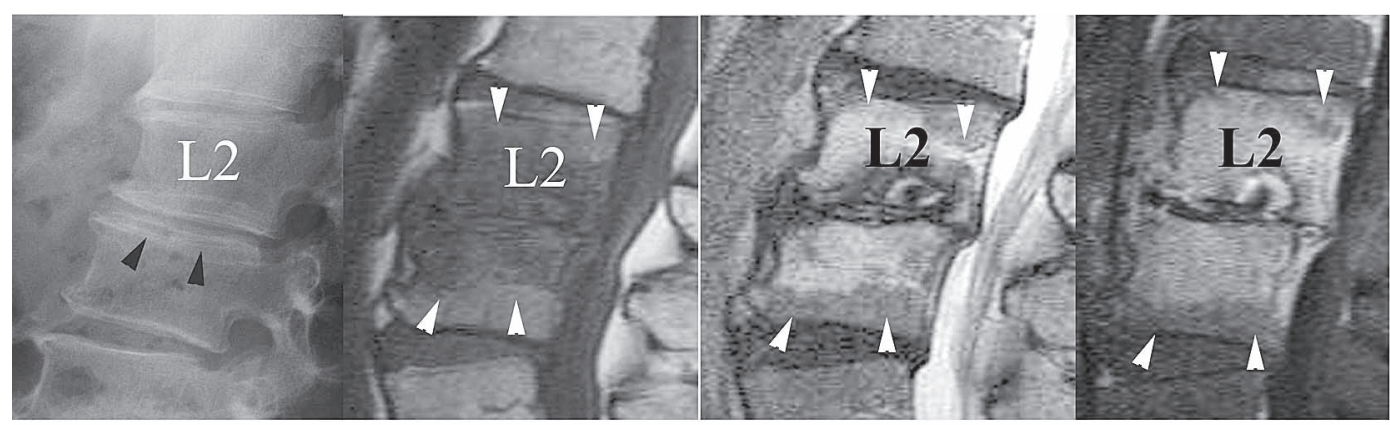

(A) (B)

(C)

(D)

Fig. 1. Typical MRI findings of pyogenic spondylitis.

A 66-year-old male; causative microorganism: unknown; and MRI: 52 days after symptomatic onset.

(A) The intervertebral disc space of L2-3 is decreasing (arrowheads), but no obvious destruction can be detected in the vertebral bodies by the plain lateral radiograph (early stage).

(B)-(D) Both L2 and L3 vertebral bodies (arrowheads) indicate low signal intensity on T1-weighted image (T1WI) (B), high intensity on T2WI (C), and are diffusely enhanced by Gadolinium-diethylenetriaminepentacetate (Gd-DTPA) (D). 


\section{Statistical Analysis}

Statistical analysis of the clinical features of the patients with typical and atypical MRI findings was performed using $t$-test, chisquare test or Mann-Whitney test by commercially available software (GraphPad Prism, GraphPad Software, San Diego, USA). A $p$ value $<0.05$ was considered statistically significant.

\section{Results}

\section{Clinical Evaluation}

The type of disease onset, and the WBC and CRP at the initial visit were summarized in Table 1 . The onset was acute or subacute in 15 patients and insidious in 7 patients according to the modified Kulowski's classification (1936). The onset was unclassified in 8 patients because their initial symptoms were paralysis, abdominal pain, acute and severe back pain without fever-up, or pleural effusion. Muscle weakness was confirmed in 8 patients and among them, a patient showed severe muscle weakness resulting in gait disability. The general condition of patients was presented in Table 2. A total of 23 patients (77\%) were immuno-compromised. Diabetes mellitus, steroid usage, and malignant diseases were most commonly noted in 6,8 and 6 patients, respectively. All 7 patients with insidious onset were immuno-compromised and 6 of these used steroids or immuno-suppressants.

Culture tests were performed in 25 patients, and the causative microorganisms were identified in 20 patients with the identification rate of $80 \%$. The causative microorganisms included methicillin-sensitive Staphylococcus

Table 1. Types of onset, White blood cell count and C-reactive protein at the initial visiting.

\begin{tabular}{lc} 
Onset (Number of Patients) & \\
Acute & 9 \\
Subacute & 6 \\
Insidious & 7 \\
Unclassified & 8 \\
White blood cell count $(/ \mu \mathrm{l})$ & $9,699(2,690-16,300)$ \\
C-reactive protein $(\mathrm{mg} / \mathrm{dl})$ & $11.9(0.1-49.8)$ \\
\hline
\end{tabular}

Table 2. General condition of the patients.

\begin{tabular}{lc}
\hline \multicolumn{1}{c}{ Complications } & Number of Patients \\
\hline Diabetes mellitus & 6 \\
Malignant disease & 6 \\
Postoperative & 2 \\
Steroid usage & 8 \\
Immunosuppressive drugs & 3 \\
Dialysis & 3 \\
Alcoholic liver dysfunction & 2 \\
Heart failure & 1 \\
None & 7 \\
\hline
\end{tabular}

(multiple choices allowed) aureus (MSSA) in 5 patients, MRSA in 3 patients, and Streptococcus mitis and Escherichia coli in 2 patients each. Chlamydia, beta-hemolytic streptococcus, methicillin-resistant Staphylococcus epidermidis (MRSE), Pseudomonas aeruginosa, MSSE, Streptococcus mutaus, Streptococcus and gram-negative bacillus were also detected in 1 patient each. Eight patients developed pyogenic spondylitis while hospitalized in other hospitals or departments. The causative microorganisms isolated from these patients included MRSA and MSSA in 2 patients each, chlamydia and Pseudomonas aeruginosa in 1 patient each, and an unknown organism in 2 patients.

\section{Radiological Evaluation}

The cervical spine was affected in 4 patients, thoracic spine in 5 patients, and lumbar spine in 23 patients. The number of affected vertebrae was most commonly 2 , which was found in 23 patients including skipped lesions in 2 patients: 1 was infected at C4-5 and L4-5 and the other was infected at T12-L1 and L3-4. Three patients had only 1 affected vertebra, whereas 2 patients had 3 consecutive affected vertebrae and 2 patients had 4 consecutive affected vertebrae. Paraspinal abscess and epidural abscess were detected in 9 and 4 patients, respectively. According to the modified Griffiths and Jones classification (1971) for plain radiographs, 21 lesions were grouped into the early stage, 8 in the destructive stage, and 3 were in the healing stage. The skipped lesions of a patient were grouped into the early stage and the healing stage, and those of another patient were both in the destructive stage.

MRI was performed at an averaged 31 days after the onset (range; 3-126 days). The MRI findings of the patients whose plain radiographs are classified into the healing stage should not indicate typical MRI findings since they reflect the absorption or resolution of the inflammation (Griffiths and Jones 1971; Modic et al. 1985). Therefore, among 29 patients whose lesions were not grouped into this stage, 13 lesions in 13 patients demonstrated typical MRI findings but 16 lesions in 16 patients did not. The atypical MRI findings could be classified into 3 categories in which a patient could show findings from 2 or more categories: 1) 9 patients did not show 2 adjacent vertebrae and the intervening intervertebral disc involvement including skipped lesions (Fig. 2); 2) 5 patients showed MRI signal changes of the lesions not involving much of the vertebral bodies but with very restricted, spotty areas involved in the bodies, or with minor signal changes of the lesions; this lesion did not clearly appear as low signal intensity on T1WI and high signal intensity on T2WI even with Gd enhancement (Fig. 3); 3 ) 3 patients had relatively larger paraspinal or epidural abscesses compared with the relatively small/minor vertebral lesions (Fig. 4). The clinical features of patients showing atypical MRI findings were compared to those showing typical MRI findings (Table 3). CRP at the initial visit in the atypical MRI group was significantly higher than that in the typical MRI group. However, there were no statistically 


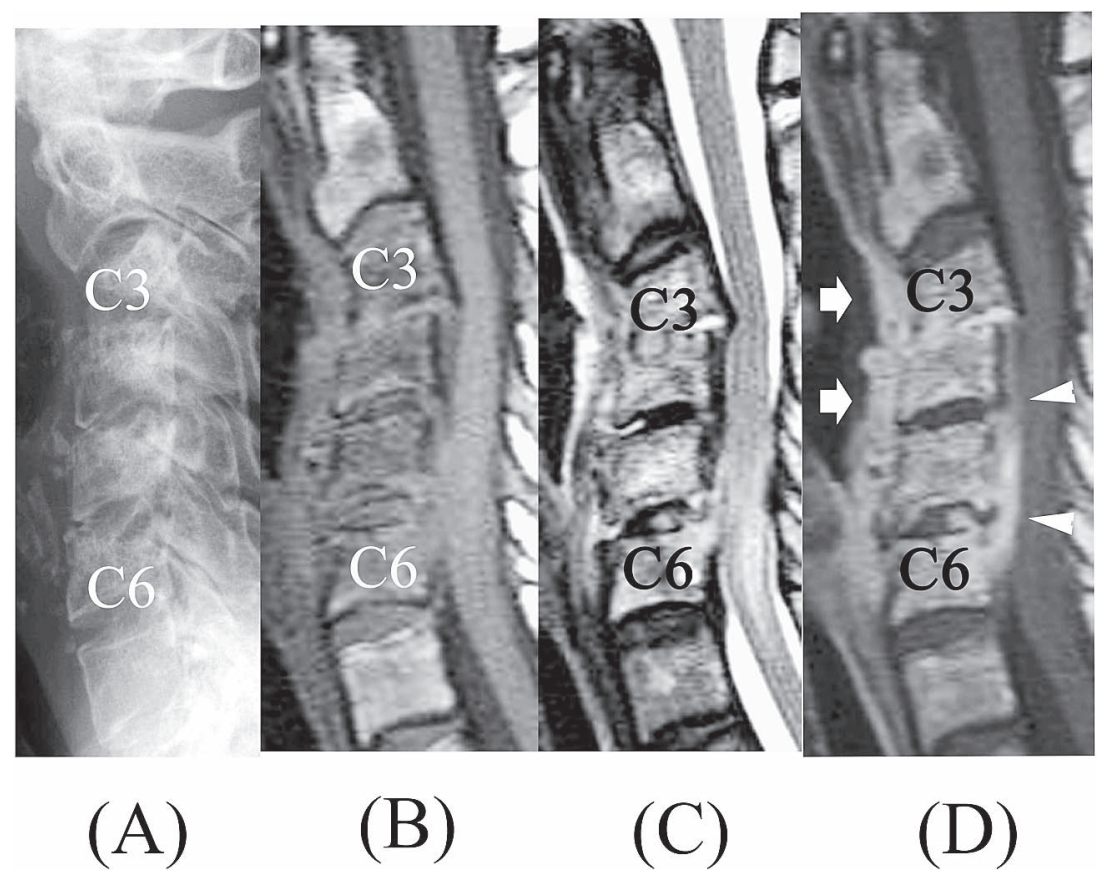

Fig. 2. Atypical MRI findings: 4 consecutive vertebrae affected.

A 36-year-old female with pharyngeal carcinoma; causative microorganism: Pseudomonas aeruginosa; and MRI: 36 days after symptomatic onset.

(A) The plain lateral radiograph demonstrates destructive changes in the C3-6 vertebral bodies and local kyphosis at C3-4 (destructive stage).

(B)-(D) The vertebral bodies from $\mathrm{C} 3$ to $\mathrm{C} 6$ show destructive change: low signal intensity on T1WI (B), high intensity on T2WI (C), and diffusely enhanced by Gd-DTPA (D). Retropharyngeal abscess (arrows) and epidural abscess (arrowheads) are also detected.

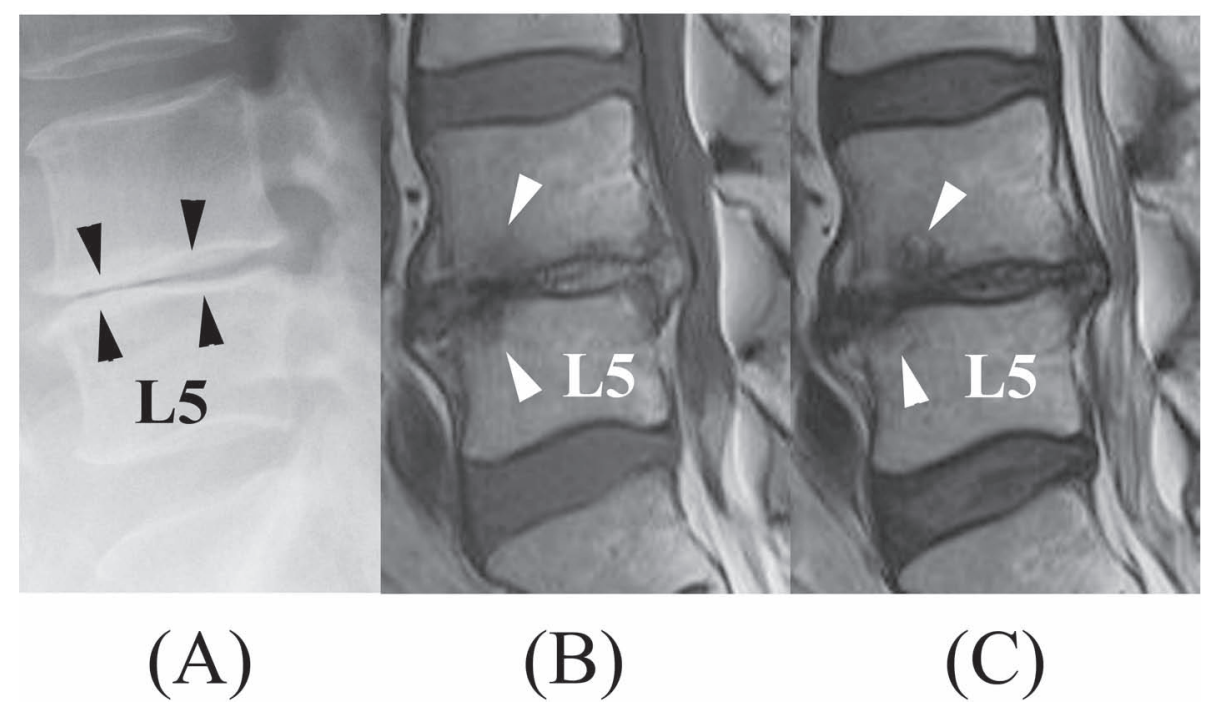

Fig. 3. Atypical MRI findings: restricted, spotty signal changes.

A 71-year-old male with diabetes mellitus, systemic lupus erythematosus and steroid use; causative microorganism: unknown; and MRI: 34 days after symptomatic onset.

(A) The plain lateral radiograph demonstrates only narrowing of the L4-5 intervertebral disc space (arrowheads) without destruction of the vertebral bodies (early stage).

(B)-(C) A very restricted part of L4 and L5 vertebral bodies (arrowheads) show low signal intensity on T1WI (B) and high on T2WI (C). 


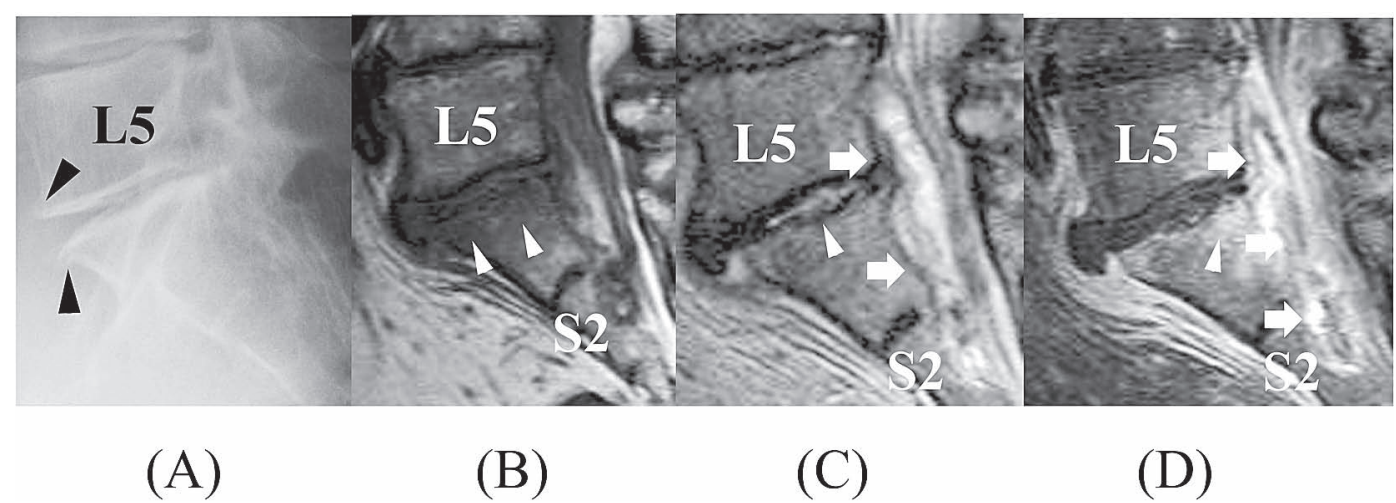

Fig. 4. Atypical MRI findings: relatively larger epidural abscess compared with the vertebral lesions.

A 72-year-old female with diabetes mellitus and steroid use; causative microorganism: Staphylococcus aureus; and MRI: 13 days after symptomatic onset.

(A) The plain lateral radiograph demonstrates only narrowing of the L5-S1 intervertebral disc space and osteophytes of the L5 vertebral body and sacrum (arrowheads) (early stage).

(B)-(D) The signal changes of T1WI and T2WI can be detected in the small area; low signal intensity lesion on T1WI is mainly detected in the S1 body (arrowheads) (B) and high on T2WI is in the L5-S1 intervertebral disc (arrowhead) and posterior part of L5 and S1 bodies that are enhanced by Gd-DTPA (arrowhead). However, large epidural abscess (arrows) is located from L5 to S2 (C, D).

Table 3. Patients' clinical features of those with typical MRI vs atypical MRI in the early and destructive stages.

\begin{tabular}{|c|c|c|c|}
\hline & Typical & Atypical & $P$ value \\
\hline Number of Patients & 13 & 16 & \\
\hline Male: Female & $8: 5$ & $7: 9$ & 0.46 \\
\hline Type of Onset* & & & 0.43 \\
\hline Acute & 2 & 6 & \\
\hline Subacute & 3 & 2 & \\
\hline Insidious & 3 & 4 & \\
\hline Unclassified & 5 & 4 & \\
\hline Plain radiographic stage** & & & 0.67 \\
\hline Early & 9 & 13 & \\
\hline Destructive & 4 & 3 & \\
\hline Age (years) (range) & $68(60-83)$ & $67(36-83)$ & 0.86 \\
\hline$\geq 80$ years & 1 & 2 & 1.00 \\
\hline White blood cell $(/ \mu 1)$ (range) & $8,782(2,690-16,600)$ & $10,420(4,600-14,300)$ & 0.37 \\
\hline C-reactive protein (mg/dl) (range) & $5.5(0.1-14.0)$ & $17.0(0.4-49.8)$ & 0.009 \\
\hline Immuno-compromised host & 12 & 11 & 0.18 \\
\hline Steroids or immuno-suppressants & 5 & 6 & 1.00 \\
\hline Interval between onset and MRI (days) (range) & $26(3-60)$ & $35(6-126)$ & 0.68 \\
\hline
\end{tabular}

* according to the modified Kulowski's classification (1936).

** according to the Griffiths and Jones classification (1971).

significant differences in any other clinical features between those 2 patient groups including the interval between the onset and MRI-examination.

\section{Discussion}

A dramatic increase in the elderly population in Japan over the past several decades because of improvements in the overall delivery of health care, including the availability of medications such as antibiotics, anticancer drugs, and immuno-suppressants and with improvements in surgical techniques has been noted. According to the 2012 world health statistics report issued by the World Heath Organization, the life expectancy for males in Japan is 80 years, whereas females live an average of 86 years. Because of the growth of this elderly population, there have also been more immuno-compromised patients in Japan. In university hospitals, many elderly patients and patients with severe general conditions have been treated. Those patients are more susceptible to infections, including pyogenic spondylitis. In Japan recently, $\geq 50 \%$ of patients with spi- 
nal infection are reported to be immuno-compromised, most frequently as a result of diabetes mellitus (Ozawa 2008). The present study also indicated that $77 \%$ of patients had chronic disorders resulting in immuno-suppression because of the use of steroids or immuno-suppressants in $37 \%$ patients and as a result of diabetes mellitus and malignancy in $20 \%$ patients each.

Typically, the onset of pyogenic spondylitis is characterized by back pain accompanied by fever (Tsiodras and Falagas 2006; Fantoni et al. 2012). However, the insidious type of onset has become more common in Japan recently, reported between 16\% and 65\% (Ozawa 2008; Yoshimoto et al. 2011). In the present study, about one-fourth of the patients showed this type of onset. Interestingly, most of them had been treated with steroids or immuno-suppressants. These drugs may inhibit the normal inflammatory response to microorganisms, which reflects in the type of onset of this disorder.

Advances in bacteriological techniques have improved the identification rate of causative microorganisms by approximately $50-60 \%$ (Ozawa 2008). The present study also demonstrated that causative microorganisms could be identified in $80 \%$ of patients who underwent tissue culture. Occasionally, patients referred to the university orthopedic departments have already been treated with antibiotics at other hospitals or departments. However, the identification rate of the causative microorganisms may not show significant differences between those who have used antibiotics before a culture test and those who have not (Tamaki et al. 2012). Bacteriological examination and culture of the infected tissues should be performed to reach a definitive diagnosis and to determine the most effective antibiotic for treatment.

It has been reported in Japan that $30-50 \%$ of patients with pyogenic spondylitis were infected with MRSA (Ozawa 2008). On the other hand, a systematic review in English literature reported that the infection rate of MRSA was only $3 \%$ of patients with this infection (Mylona et al. 2008). In the present study, we showed that $16 \%$ of the patients with this disorder (4 out of 25 patients) were infected by MRSA or MRSE. Taken together, these results suggest that the rate of pyogenic spondylitis may be higher in Japan. MRSA infection is significantly associated with immuno-compromised hosts, longer hospitalizations before infection, and longer antibiotic therapy (Tacconelli et al. 2008; Yamada et al. 2011; Sasaji et al. 2012). According to the data from the Organization for Economic Cooperation and Development (OECD) in 2007, the average days of hospitalization in Japan was 35.7 , whereas in the United States of America the average was 6.5, in the United Kingdom it was 7.0. In addition in Japan, once a patient develops fever-up, antibiotics are often prescribed without specifically discerning the cause of the fever. Such uncontrolled use of antibiotics can produce antibiotic-resistant mutated microorganisms (Tacconelli et al. 2008). Longer hospitalization and uncontrolled use of antibiotics in Japan may result in high infection rates with methicillin-resistant staphylococci leading to spondylitis (Yoshimoto et al. 2011; Sasaji et al. 2012).

MRI has a high sensitivity and specificity to detect early infection, to evaluate fully the extent of disease, and to monitor the therapeutic response during the course of spinal infection (Tsiodras and Falagas 2006). The typical MRI features of active pyogenic spondylitis include a low signal intensity on T1WI, and a high signal intensity on T2WI with clear enhancement by Gd contrast in a spinal motion segment, including much of the 2 continuous vertebral bodies and an intervertebral disc between them (Modic et al. 1985; An and Seldomridge 2006; DeSanto and Ross 2011; Cheung and Luk 2012; Leone et al. 2012). Previous studies conducted in the 1980s and 1990s indicated that approximately $90 \%$ of patients with pyogenic spondylitis demonstrated such typical MRI findings (Modic et al. 1985; Dagirmanjian et al. 1996). However, in the present study, more than half of the patients did not show typical, classical MRI findings even though they showed the early or destructive stages on plain radiographcal findings.

The reasons why more than half of the patients with active pyogenic spondylitis showed atypical MRI findings were uncertain. The MRI findings in the present study showed no obvious relationship with regard to the time interval between the onset of symptoms and the date of the MRI examination. However, the exact onset of pyogenic spondylitis may not correspond with the onset of the symptoms either. It may also be difficult to definitely confirm the symptomatic onset of the subacute and insidious types. In addition, each stage of the plain radiologic classification is within a certain time period. In the early stage itself, some patients may be diagnosed at relatively early after the onset while others may be diagnosed close to the destructive stage. Therefore, it would be extremely difficult to compare the MRI findings recorded at actually same time interval after the exact onset, which is one of the limitations of this kind of radiographic studies.

Another possibility was that several drugs such as steroids, immuno-suppressants, and anticancer drugs affected the normal progression of disease. Although there were no significant differences in the rate of immuno-compromised patients and the usage of such drugs between patients showing typical and atypical MRI findings, inflammatory reaction and infectious disease progression may be modified by the immunologic condition of the individual patient, which may have been affected by those kinds of drugs. Accordingly, MRI may not demonstrate typical findings.

In addition to a relatively small sample size and the aforementioned limitation, the data in the present study were retrospectively obtained; therefore, the clinical features could be only roughly assessed from medical records. However, it does indicate several features of pyogenic spondylitis as observed recently in university hospitals in Japan. The fact that about half of the patients did not demonstrate typical MRI findings is interesting. Because of the 
demographic shift with an aging population, and with an increasing number of immuno-compromised patients, this result may become more common in Japan in the near future, not only in university hospitals but also in other hospitals or clinics.

\section{Conflict of Interest}

The authors declare no conflict of interest.

\section{References}

An, H.S. \& Seldomridge, J.A. (2006) Spinal infections: diagnostic tests and imaging studies. Clin. Orthop. Relat. Res., 444, 2733.

Carragee, E.J. (1997) The clinical use of magnetic resonance imaging in pyogenic vertebral osteomyelitis. Spine, 22, 780-785.

Cheung, W.Y. \& Luk, K.D.K. (2012) Pyogenic spondylitis. Int. Orthop. (SICOT), 36, 397-404.

Dagirmanjian, A., Schils, J., McHenry, M. \& Modic, M.T. (1996) MR imaging of vertebral osteomyelitis revisited. AJR Am. J. Roentgenol., 167, 1539-1543.

DeSanto, J. \& Ross, J.S. (2011) Spine infection/inflammation. Radiol. Clin. North Am., 49, 105-127.

Fantoni, M., Trecarichi, E.M., Rossi, B., Mazzotta, V., Di Giacomo, G., Nasto, L.A., Di Meco, E. \& Pola, E. (2012) Epidemiological and clinical features of pyogenic spondylodiscitis. Eur. Rev. Med. Pharmacol. Sci., 16 (Suppl 2), 2-7.

Fukuda, K., Miyamoto, H., Uno, K. \& Okada, Y. (2012) Indications and limitations of conservative treatment for pyogenic spondylitis. J. Spinal Disord. Tech., [Epub ahead of print].

Griffiths, H.E.D. \& Jones, D.M. (1971) Pyogenic infection of the spine. A review of twenty-eight cases. J. Bone Joint Surg. Br., 53, 383-391.

Kim, C.W., Currier, B.L. \& Eismont, F.J. (2011) Infection of the spine. In Rothman-Simeone The Spine, 6th ed., edited by Herkowitz, H.N., Garfin, S.R., Eismont, F.J., Bell, G.R. \& Balderston, R.A. Elsevier Inc., Philadelphia, pp. 1513-1570.

Kulowski, J. (1936) Pyogenic osteomyelitis of the spine. An analysis and discussion of 102 cases. J. Bone Joint Surg. Am., 18, 343-364.
Leone, A., Dell'Atti, C., Magarelli, N., Colelli, P., Balanika, A., Casale, R. \& Bonomo, L. (2012) Imaging of spondylodiscitis. Eur. Rev. Med. Pharmacol. Sci., 16 (Suppl 2), 8-19.

Modic, M.T., Feiglin, D.H., Piraino, D.W., Boumphrey, F., Weinstein, M.A., Duchesneau, P. \& Rehm, S. (1985) Vertebral osteomyelitis: assessment using MR. Radiology, 157, 157-166.

Mylona, E., Samarkos, M., Kakalou, E., Fanourgiakis, P. \& Skoutelis, A. (2008) Pyogenic vertebral osteomyelitis: a systematic review of clinical characteristics. Semin. Arthritis Rheum., 39, 10-17.

Ozawa, H. (2008) Epidemiology of pyogenic spondylitis. SekituiSekizui, 21, 1084-1090 (in Japanese).

Sasaji, T., Yamada, N. \& Iwai, K. (2012) Microorganisms causing pyogenic spondylitis: comparison of community and hospitalacquired types. Ups. J. Med. Sci., 117, 399-401.

Tacconelli, E., De Angelis, G., Cataldo, M.A., Pozzi, E. \& Cauda, R. (2008) Does antibiotic exposure increase the risk of methicillin-resistant Staphylococcus aureus (MRSA) isolation? A systematic review and meta-analysis. J. Antimicrob. Chemother., 61, 26-38.

Tamaki, Y., Hyakuna, K. \& Uchikoshi, A. (2012) Analysis of culture of pyogenic spondylitis. J. Spine Res., 3, 29-32 (in Japanese)

Tsiodras, S. \& Falagas, M.E. (2006) Clinical assessment and medical treatment of spine infections. Clin. Orthop. Relat. Res., 444, 38-50.

Washio, M., Kiyohara, C., Hamada, T., Miyake, Y., Arai, Y. \& Okayama, M. (1997) The case fatality rate of methicillinresistant Staphylococcus aureus (MRSA) infection among the elderly in a geriatric hospital and their risk factors. Tohoku J. Exp. Med., 183, 75-82.

Yamada, K., Yanagihara, K., Hara, Y., Araki, N., Harada, Y., Morinaga, Y., Matsuda, J., Izumikawa, K., Seki, M., Kakeya, H., Yamamoto, Y., Kohno, S. \& Kamihira, S. (2011) Clinical features of bacteremia caused by methicillin-resistant Staphylococcus aureus in a tertiary hospital. Tohoku J. Exp. Med., 224, 61-67.

Yoshimoto, M., Takebayashi, T., Kawaguchi, S., Tsuda, H., Ida, K., Wada, T. \& Yamashita, T. (2011) Pyogenic spondylitis in the elderly: a report from Japan with the most aging society. Eur. Spine J., 20, 649-654. 